\title{
AN INTERPOLATION PROBLEM FOR FUNCTIONS WITH VALUES IN A COMMUTATIVE RING
}

\author{
DANIEL ALPAY AND HAIM ATTIA \\ Dedicated to the memory of Israel Gohberg
}

\begin{abstract}
It was recently shown that the theory of linear stochastic systems can be viewed as a particular case of the theory of linear systems on a certain commutative ring of power series in a countable number of variables. In the present work we study an interpolation problem in this setting. A key tool is the principle of permanence of algebraic identities.
\end{abstract}

\section{Contents}

1. Introduction

2. The white noise space

3. The ring $\mathfrak{R}$

4. Analytic functions with values in $\mathfrak{R}$

5. Nevanlinna-Pick Interpolation 14

6. More general interpolation problem 17

References 18

\section{INTRODUCTION}

There are numerous connections between classical interpolation problems and optimal control and the theory of linear systems; see for instance [10, 1]. In these settings, the coefficient space is the complex field $\mathbb{C}$, or in the case of real systems, the real numbers $\mathbb{R}$. Furthermore, already from its inception, linear system theory was considered when the coefficient space is a general (commutative) field, or more generally a commutative ring; see [22, 25]. In [8, 6] a new approach

1991 Mathematics Subject Classification. 60H40, 93C05.

Key words and phrases. white noise space, stochastic distributions, linear systems on rings.

D. Alpay thanks the Earl Katz family for endowing the chair which supported his research. 
to the theory of linear stochastic systems was developed, in which the coefficient space is now a certain commutative ring $\mathfrak{R}$ (see Section 3 below). The results from [22, 25] do not seem to be directly applicable to this theory, and the specific properties of $\mathfrak{R}$ played a key role in the argumemts in [8, 6].

The purpose of this work is to discuss the counterparts of classical interpolation problems in this new setting. To set the problems into perspective, we begin this introduction with a short discussion of the deterministic case. In the classical theory of linear systems, inputoutput relations of the form

$$
y_{n}=\sum_{k=0}^{n} h_{n-k} u_{k}, \quad n=0,1, \ldots
$$

where $\left(u_{n}\right)_{n \in \mathbb{N}_{0}}$ is called the input sequence, $\left(y_{n}\right)_{n \in \mathbb{N}_{0}}$ is the output sequence, and $\left(h_{n}\right)_{n \in \mathbb{N}_{0}}$ is the impulse response, play an important role. The sequence $\left(h_{n}\right)_{n \in \mathbb{N}_{0}}$ may consist of matrices (of common dimensions), and then the input and output sequences consist of vectors of appropriate dimensions. Similarly state space equations

$$
\begin{aligned}
x_{n+1} & =A x_{n}+B u_{n}, \\
y_{n} & =C x_{n}+D u_{n}, \quad n=0,1, \ldots
\end{aligned}
$$

play an important role. Here $x_{n}$ denotes the state at time $n$, and $A, B, C$ and $D$ are matrices with complex entries. The transfer function of the system is

$$
h(\lambda)=\sum_{n=0}^{\infty} h_{n} \lambda^{n}
$$

in the case (1.1), and

$$
h(\lambda)=D+\lambda C(I-\lambda A)^{-1} B
$$

in the case of state space equations, when assuming the state at $n=0$ to be equal to 0 . Classical interpolation problems bear various applications to the corresponding linear systems. See for instance [10, Part VI], 21]. To fix ideas, we consider the case of bitangential interpolation problem for matrix-valued functions analytic and contractive in the open unit disk (Schur functions), and will even consider only the Nevanlinna-Pick interpolation problem in the sequel to keep notation simple, but it will be clear that the discussion extends to more general cases. Recall (see [10, $\S 18.5$ p. 409]) that the bitangential interpolation problem may be defined in terms of a septuple of matrices 
$\omega=\left(C_{+}, C_{-}, A_{\pi}, A_{\zeta}, B_{+}, B_{-}, \Gamma\right)$ by the conditions

$$
\begin{aligned}
\sum_{\lambda_{0} \in \mathbb{D}} \operatorname{Res}_{\lambda=\lambda_{0}}\left(\lambda I-A_{\zeta}\right)^{-1} B_{+} S(\lambda) & =-B_{-}, \\
\sum_{\lambda_{0} \in \mathbb{D}} \operatorname{Res}_{\lambda=\lambda_{0}} S(\lambda) C_{-}\left(\lambda I-A_{\pi}\right)^{-1} & =C_{+}, \\
\sum_{\lambda_{0} \in \mathbb{D}} \operatorname{Res}_{\lambda=\lambda_{0}}\left(\lambda I-A_{\zeta}\right)^{-1} B_{+} S(\lambda) C_{-}\left(\lambda I-A_{\pi}\right)^{-1} & =\Gamma,
\end{aligned}
$$

where $A_{\zeta}$ and $A_{\pi}$ have their spectra in the open unit disk, where $\left(A_{\zeta}, B_{+}\right)$is a full range pair (that is, controllable) and where $\left(C_{-}, A_{\pi}\right)$ is a null kernel pair (that is, observable). We send the reader to [10] for the definitions. Moreover, $\Gamma$ satisfies moreover the compatibility condition

$$
\Gamma A_{\pi}-A_{\zeta} \Gamma=B_{+} C_{+}+B_{-} C_{-} .
$$

Let $P$ be the matrix (see [10, p. 458])

$$
P=\left(\begin{array}{cc}
P_{1} & \Gamma^{*} \\
\Gamma & P_{2}
\end{array}\right)
$$

where $P_{1}$ and $P_{2}$ are the solutions of the Stein equations

$$
\begin{aligned}
P_{1}-A_{\pi}^{*} P_{1} A_{\pi} & =C_{-}^{*} C_{-}-C_{+}^{*} C_{+} \\
P_{2}-A_{\zeta} P_{2} A_{\zeta}^{*} & =B_{+} B_{+}^{*}-B_{-} B_{-}^{*} .
\end{aligned}
$$

Furthermore, and assuming the unknown function $S$ to be $\mathbb{C}^{p \times q}$-valued,

$$
J=\left(\begin{array}{cc}
I_{p} & 0 \\
0 & -I_{q}
\end{array}\right) .
$$

When $P$ is strictly positive, the solutions of the interpolation problem are given in terms of a linear fractional transformation based on a $J$ inner rational function $\Theta$ built from the septuple $\omega$ via the formula (see [10, (18.5.6) p. 410])

$$
\begin{aligned}
\Theta(\lambda)=I+ & \left(\lambda-\lambda_{0}\right)\left(\begin{array}{cc}
C_{+} & -B_{+}^{*} \\
C_{-} & B_{-}^{*}
\end{array}\right)\left(\begin{array}{cc}
\left(\lambda I-A_{\pi}\right)^{-1} & 0 \\
0 & \left(I-\lambda A_{\zeta}^{*}\right)^{-1}
\end{array}\right) \times \\
(1.3) & \times P^{-1}\left(\begin{array}{cc}
\left(I-\lambda_{0} A_{\pi}^{*}\right)^{-1} C_{+}^{*} & -\left(I-\lambda_{0} A_{\pi}^{*}\right)^{-1} C_{-}^{*} \\
\left(A_{\zeta}-\lambda_{0} I\right)^{-1} B_{+} & \left(A_{\zeta}-\lambda_{0} I\right)^{-1} B_{-}
\end{array}\right)
\end{aligned}
$$

where $\lambda_{0}$ is fixed on the unit circle and such that the various inverses exist in the above formula. An important fact is that the entries of $P_{1}$ and $P_{2}$ are rational functions of the entries of the matrices of $\omega$. As a consequence, there exists a rational function $f(\lambda)$, built from $\omega$ and such that the entries of $\Theta$ are polynomials in $\lambda$, with coefficients which 
are themselves polynomials in the entries of the matrices of $\omega$ with coefficients in $\mathbb{Z}$. This fact will allow us in the sequel to use the principle of permanence of identities (see [9, p. 456]), to extend interpolation problem to a more general setting.

Allowing in (1.1) the input sequence $\left(u_{n}\right)_{n \in \mathbb{N}_{0}}$ to consist of random variables has been considered for a long time. On the other hand, allowing also the impulse response of the system to carry some randomness seems much more difficult to tackle. Recently a new approach to the theory of linear stochastic systems was developed using Hida's white noise space theory [18], [19], 23], and Kondratiev's spaces of stochastic test functions and distributions [20. In this approach, see [3], [5], 6], the complex numbers are replaced by random variables in the white noise space, or more generally, by stochastic distributions in the Kondratiev space, and the product of complex numbers is replaced by the Wick product. For instance, (1.1) now becomes

$$
y_{n}=\sum_{k=0}^{n} h_{n-k} \diamond u_{k}, \quad n=0,1, \ldots
$$

where the various quantities are in the white noise space, or more generally in the Kondratiev's space of stochastic distributions, and $\diamond$ denotes the Wick product. An important role in this theory is played by a ring $\mathfrak{R}$ of power series in countably many variables with coefficients in $\mathbb{C}$. This ring is endowed with a topology, which is that of the dual of a countably normed nuclear space. See Sections 2 and 3. Let us denote by

$$
\mathbf{r}(z)=\sum_{\alpha \in \ell} r_{\alpha} z^{\alpha}
$$

an element of $\mathfrak{R}$, where $\ell$ denotes the set of sequences $\left(\alpha_{1}, \alpha_{2}, \ldots\right)$, whose entries are in

$$
\mathbb{N}_{0}=\{0,1,2,3, \ldots\},
$$

and for which $\alpha_{k} \neq 0$ for only a finite number of indices $k$, and where we have used the multi-index notation

$$
z^{\alpha}=z_{1}^{\alpha_{1}} z_{2}^{\alpha_{2}} \cdots \quad \alpha \in \ell .
$$

The ring $\mathfrak{R}$ has the following properties:

$\left(P_{1}\right)$ If $\mathbf{r} \in \mathfrak{R}$ and $\mathbf{r}(0,0,0, \cdots) \neq 0$, then $\mathbf{r}$ has an inverse in $\mathfrak{R}$.

$\left(P_{2}\right)$ If $\mathbf{r} \in \mathfrak{R}^{n \times n}$ is such that $\mathbf{r}(0,0,0, \cdots)=0_{n \times n}$ and if $f$ is a function of one complex variable, analytic in a neighborhood of the origin, with 
Taylor expansion

$$
f(\lambda)=\sum_{p=0}^{\infty} f_{p} \lambda^{p}
$$

then, the series

$$
f(\mathbf{r}) \stackrel{\text { def. }}{=} \sum_{p=0}^{\infty} f_{p} \mathbf{r}^{p}
$$

converges in $\mathfrak{R}^{n \times n}$. Furthermore, if $g$ is another function of one complex variable, analytic in a neighborhood of the origin, we have

$$
(f g)(\mathbf{r})=f(\mathbf{r}) g(\mathbf{r}) .
$$

$\left(P_{3}\right)$ If $\mathbf{r}(z)=\sum_{\alpha \in \ell} r_{\alpha} z^{\alpha} \in \mathfrak{R}$, then $\mathbf{r}^{*}(z) \stackrel{\text { def. }}{=} \sum_{\alpha \in \ell} r_{\alpha}^{*} z^{\alpha} \in \mathfrak{R}$, where $r_{\alpha}^{*}$ denotes the conjugate of the complex number $r_{\alpha}$.

Property $\left(P_{1}\right)$ implies in particular that a matrix $\mathbf{A} \in \mathfrak{R}^{n \times n}$ is invertible in $\mathfrak{R}^{n \times n}$ if and only if $\operatorname{det} \mathbf{A}(0) \neq 0$. This fact, together with $\left(P_{2}\right)$, allows to define expressions such as

$$
\mathscr{H}(\lambda)=\mathbf{D}+\lambda \mathbf{C}\left(I_{n}-\lambda \mathbf{A}\right)^{-1} \mathbf{B}=\mathbf{D}+\sum_{k=1}^{\infty} \lambda^{k} \mathbf{C A}^{k-1} \mathbf{B},
$$

where $\mathbf{A}, \mathbf{B}, \mathbf{C}$, and $\mathbf{D}$ are matrices of appropriate dimensions and with entries in $\mathfrak{R}$, and where $\lambda$ is an independent complex variable. As explained in [6] this is the transfer function of some underlying linear systems, and is a rational function with coefficients in $\mathfrak{R}$.

The purpose of this paper is to explain how to tackle in the present setting counterparts of some classical interpolation problems which appear in the theory of linear systems. To illustrate our strategy, we focus on the Nevanlinna-Pick interpolation problem, but our method works the same for the general bitangential interpolation problem. The computations done in the classical theory (that is, when the coefficient space consists of the complex numbers) extend to the case where $\mathbb{C}$ is replaced by the ring $\mathfrak{R}$. In some cases, such as Nevanlinna-Pick interpolation, this can be shown by direct computations. In the general case, one needs to use the principle of permanence of identities, see [9, p. 456]. We note that there are other commutative rings with properties $\left(P_{1}\right),\left(P_{2}\right)$ and $\left(P_{3}\right)$ for which the above analysis is applicable. See [7].

The paper consists of six sections besides the present introduction. In the second section we review Hida's white noise space setting and the 
Kondratiev spaces of stochastic distributions. The definition and main properties of the ring $\mathfrak{R}$ are given in Section 3. In Section 4 we define and study analytic functions from an open set of $\mathbb{C}$ with values in $\mathfrak{R}$. In Section 5 we consider the Nevanlinna-Pick interpolation problem. In the last section we discuss the bitangential interpolation problem.

\section{The White NOISE SPACE}

We here review Hida's white noise space theory and the associated spaces of stochastic distributions introduced by Kondratiev. See [18], [19], [20], [23]. Let $\mathscr{S}(\mathbb{R})$ denote the Schwartz space of smooth realvalued rapidly decreasing functions. It is a nuclear space, and by the Bochner-Minlos theorem (see [15, Théorème 2, p. 342]), there exists a

probability measure on the Borel sets $\mathcal{B}$ of the dual space $\mathscr{S}(\mathbb{R})^{\prime} \stackrel{\text { def. }}{=} \Omega$ such that

$$
e^{-\frac{\|s\|_{\mathbf{L}_{2}(\mathbb{R})}^{2}}{2}}=\int_{\Omega} e^{i\langle\omega, s\rangle} d P(\omega), \quad \forall s \in \mathscr{S}(\mathbb{R}),
$$

where the brackets $\langle\cdot, \cdot\rangle$ denote the duality between $\mathscr{S}(\mathbb{R})$ and $\mathscr{S}^{\prime}(\mathbb{R})$. The probability space

$$
\mathcal{W}=(\Omega, \mathcal{B}, d P)
$$

is called the white noise probability space. We will be interested in particular in $\mathbf{L}_{2}(\mathcal{W})$, called the white noise space. For $s \in \mathscr{S}(\mathbb{R})$, let $Q_{s}$ denote the random variable

$$
Q_{s}(\omega)=\langle\omega, s\rangle .
$$

It follows from (2.8) that

$$
\|s\|_{\mathbf{L}_{2}(\mathbb{R})}=\left\|Q_{s}\right\|_{\mathbf{L}_{2}(\mathcal{W})} .
$$

Therefore, $Q_{s}$ extends continuously to an isometry from $\mathbf{L}_{2}(\mathbb{R})$ into $\mathbf{L}_{2}(\mathcal{W})$. In the presentation of the Gelfand triple associated to the white noise space which we will use, we follow [20]. The white noise space $\mathbf{L}_{2}(\mathcal{W})$ admits a special orthogonal basis $\left(H_{\alpha}\right)_{\alpha \in \ell}$, indexed by the set $\ell$ and built in terms of the Hermite functions $\widetilde{h}_{k}$ and of the Hermite polynomials $h_{k}$ defined by

$$
H_{\alpha}(\omega)=\prod_{k=1}^{\infty} h_{\alpha_{k}}\left(Q_{\widetilde{h}_{k}}(\omega)\right)
$$

We refer to [20, Definition 2.2.1 p. 19] for more information. In terms of this basis, any element of $\mathbf{L}_{2}(\mathcal{W})$ can be written as

$$
F=\sum_{\alpha \in \ell} f_{\alpha} H_{\alpha}, \quad f_{\alpha} \in \mathbb{C},
$$


with

$$
\|F\|_{\mathcal{W}}^{2}=\sum_{\alpha \in \ell}\left|f_{\alpha}\right|^{2} \alpha !<\infty
$$

There are quite a number of Gelfand triples associated to $\mathbf{L}_{2}(\mathcal{W})$. In our previous works [2], [5], and in the present one, we focus on the one consisting of the Kondratiev space $S_{1}$ of stochastic test functions, of $\mathcal{W}$, and of the Kondratiev space $S_{-1}$ of stochastic distributions. To define these spaces we first introduce for $k \in \mathbb{N}$ the Hilbert space $\mathcal{H}_{k}$ which consists of series of the form (1.5) such that

$$
\|F\|_{k} \stackrel{\text { def. }}{=}\left(\sum_{\alpha \in \ell}(\alpha !)^{2}\left|f_{\alpha}\right|^{2}(2 \mathbb{N})^{k \alpha}\right)^{1 / 2}<\infty,
$$

and the Hilbert spaces $\mathcal{H}_{k}^{\prime}$ consisting of sequences $\left(f_{\alpha}\right)_{\alpha \in \ell}$ such that

$$
\|F\|_{k}^{\prime} \stackrel{\text { def. }}{=}\left(\sum_{\alpha \in \ell}\left|f_{\alpha}\right|^{2}(2 \mathbb{N})^{-k \alpha}\right)^{1 / 2}<\infty .
$$

We note that, for $F \in \mathcal{H}_{k}^{\prime}$ we have

$$
\lim _{\substack{p \geq k \\ p \rightarrow \infty}}\|F\|_{p}^{\prime}=\left|f_{(0,0,0 \ldots)}\right|^{2}
$$

as can be seen, for instance, by applying the dominated convergence theorem to an appropriate discrete measure. Following the usage in the literature, we will also write the elements of $\mathcal{H}_{k}^{\prime}$ as formal power series $\sum_{\alpha \in \ell} f_{\alpha} H_{\alpha}$. Note that $\left(\mathcal{H}_{k}\right)_{k \in \mathbb{N}}$ forms a decreasing sequence of Hilbert spaces, with increasing norms, while $\left(\mathcal{H}_{k}^{\prime}\right)_{k \in \mathbb{N}}$ forms an increasing sequence of Hilbert spaces, with decreasing norms. The spaces $S_{1}$ and $S_{-1}$ are defined by the corresponding projective and inductive limits

$$
S_{1}=\bigcap_{k=1}^{\infty} \mathcal{H}_{k} \quad \text { and } \quad S_{-1}=\bigcup_{k=1}^{\infty} \mathcal{H}_{k}^{\prime} .
$$

The Wick product is defined on the basis $\left(H_{\alpha}\right)_{\alpha \in \ell}$ by

$$
H_{\alpha} \diamond H_{\beta}=H_{\alpha+\beta} .
$$

It extends to an everywhere defined and continuous map from $S_{1} \times S_{1}$ into itself and from $S_{-1} \times S_{-1}$ into itself Let $l>0$, and let $k>l+1$. Consider $h \in \mathcal{H}_{l}^{\prime}$ and $u \in \mathcal{H}_{k}^{\prime}$. Then, Våge's inequality holds:

$$
\|h \diamond u\|_{k}^{\prime} \leq A(k-l)\|h\|_{l}^{\prime}\|u\|_{k}^{\prime}
$$

\footnotetext{
${ }^{1}$ The continuity properties are proved in [7] for a more general family of rings.
} 
where

$$
A(k-l)=\left(\sum_{\alpha \in \ell}(2 \mathbb{N})^{(l-k) \alpha}\right)^{1 / 2}<\infty .
$$

See [20, Proposition 3.3.2 p. 118]. The following result is a direct consequence of (2.13) and will be useful in the sequel.

Lemma 2.1. Let $F \in \mathcal{H}_{p}^{\prime}$. Then, $F^{\diamond n} \stackrel{\text { def. }}{=} \underbrace{F \diamond \cdots \diamond F}_{n \text { times }} \in \mathcal{H}_{p+2}^{\prime}$ and

$$
\left\|F^{\diamond n}\right\|_{p+2}^{\prime} \leq \frac{1}{A(2)}\left(A(2)\|F\|_{p}^{\prime}\right)^{n}, \quad n=1,2,3 \ldots
$$

Proof: We proceed by induction. The case $n=1$ holds since

$$
\|F\|_{p+2}^{\prime} \leq\|F\|_{p}^{\prime}, \quad \text { for } \quad F \in \mathcal{H}_{p}^{\prime} .
$$

Assume now that (2.14) holds at rank $n$. Then, from (2.12) we have

$$
\begin{aligned}
\left\|F^{\diamond(n+1)}\right\|_{p+2}^{\prime} & \leq A(2)\|F\|_{p}^{\prime}\left\|F^{\diamond n}\right\|_{p+2}^{\prime} \\
& \leq A(2))\|F\|_{p}^{\prime} \frac{1}{A(2)}\left(A(2)\|F\|_{p}^{\prime}\right)^{n} \\
& =\frac{1}{A(2)}\left(A(2)\|F\|_{p}^{\prime}\right)^{n+1} .
\end{aligned}
$$

\section{THE RING $\mathfrak{R}$}

The Kondratiev space $S_{-1}$ endowed with the Wick product is a commutative ring of sequences $\left(c_{\alpha}\right)_{\alpha \in \ell}$, with properties $\left(P_{1}\right),\left(P_{2}\right)$ and $\left(P_{3}\right)$, where in $\left(P_{1}\right)$ one understands by evaluation at the origin the first coefficient of the sequence. Using the Hermite transform (defined below), we view $S_{-1}$ as a ring of powers series in infinitely many variables. We point out that there are other commutative rings of sequences with properties $\left(P_{1}\right),\left(P_{2}\right)$ and $\left(P_{3}\right)$, and for which a counterpart of the Hermite transform holds. See [7].

The Hermite transform is defined by

$$
I\left(H_{\alpha}\right)=z^{\alpha}, \quad \text { with } \alpha \in \ell \quad \text { and } z=\left(z_{1}, z_{2}, \ldots\right) \in \mathbb{C}^{\mathbb{N}} .
$$

Then

$$
I\left(H_{\alpha} \diamond H_{\beta}\right)=I\left(H_{\alpha}\right) I\left(H_{\beta}\right) .
$$


It extends for $F=\sum_{\alpha \in \ell} a_{\alpha} H_{\alpha} \in S_{-1}$ by the formula $I(F)(z)=$ $\sum_{\alpha \in \ell} a_{\alpha} z^{\alpha}$, and converges in sets of the form

$$
K_{p}(R)=\left\{z \in \mathbb{C}^{\mathbb{N}}: \sum_{\alpha \neq 0}|z|^{\alpha}(2 \mathbb{N})^{p \alpha}<R^{2}\right\},
$$

where $p$ is such that $F \in \mathcal{H}_{p}^{\prime}$. The Kondratiev space $S_{-1}$ is closed under the Wick product, and we have

$I(F \diamond G)(z)=I(F)(z) I(G)(z) \quad$ and $\quad I(F+G)(z)=I(F)(z)+I(G)(z)$

for any $F, G \in S_{-1}$. Therefore the image of the Kondratiev space $S_{-1}$ under the Hermite transform is a commutative ring, denoted by

$$
\mathfrak{R} \stackrel{\text { def. }}{=} \operatorname{Im}\left(\mathrm{I}\left(\mathrm{S}_{-1}\right)\right) \text {. }
$$

This ring was introduced in [6]. We transpose to it via the Hermite transform the properties of $S_{-1}$. We have

$$
\mathfrak{R}=\bigcup_{k=1}^{\infty} I\left(\mathcal{H}_{k}^{\prime}\right) .
$$

We define the adjoint $\mathbf{G}^{*}=\left(\mathbf{h}_{s t}\right) \in \mathfrak{R}^{m \times n}$ of $\mathbf{G}=\left(\mathbf{g}_{t s}\right) \in \mathfrak{R}^{n \times m}$ by $\mathbf{h}_{s t}(z)=\mathbf{g}_{t s}^{*}(z)(t \in\{1, \ldots, n\}$ and $s \in\{1, \ldots, m\})$. Then for $\mathbf{A} \in \mathfrak{R}^{n \times m}$ and $\mathbf{B} \in \mathfrak{R}^{m \times u}$ we have

$$
(\mathrm{AB})^{*}=\mathbf{B}^{*} \mathbf{A}^{*} \text {. }
$$

Note that $\mathbf{G}^{*}(0)=\mathbf{G}(0)^{*}$, where $\mathbf{G}(0)^{*}$ is the usual adjoint matrix.

Definition 3.1. An element $\mathbf{A} \in \mathfrak{R}^{n \times n}$ will be said strictly positive, $\mathbf{A}>0$, if it can be written as $\mathbf{A}=\mathbf{G G}^{*}$, where $\mathbf{G} \in \mathfrak{R}^{n \times n}$ is invertible. It will be said positive if $\mathbf{G}$ is not assumed to be invertible.

Lemma 3.2. Let $\mathbf{A} \in \mathfrak{R}^{n \times n}$. Then, $\mathbf{A}$ is strictly positive if and only if $\mathbf{A}(0) \in \mathbb{C}^{n \times n}$ is a strictly positive matrix (in the usual sense).

Proof: If $\mathbf{A}=\mathbf{G G}^{*}$ with $\operatorname{det} \mathbf{G}(0) \neq 0$, then $\mathbf{A}(0)=\mathbf{G}(0) \mathbf{G}(0)^{*}$ is a strictly positive matrix. Conversely, assume that $\mathbf{A} \in \mathfrak{R}^{n \times n}$ is such that $\mathbf{A}(0)>0$. We write

$$
\begin{aligned}
\mathbf{A}(z) & =\mathbf{A}(0)+(\mathbf{A}(z)-\mathbf{A}(0)) \\
& =\sqrt{\mathbf{A}(0)}\left\{I_{n}+(\sqrt{\mathbf{A}(0)})^{-1}(\mathbf{A}(z)-\mathbf{A}(0))(\sqrt{\mathbf{A}(0)})^{-1}\right\} \sqrt{\mathbf{A}(0)} .
\end{aligned}
$$

Let $\mathbf{E}(z)=\sqrt{\mathbf{A}(0)})^{-1}(\mathbf{A}(z)-\mathbf{A}(0))(\sqrt{\mathbf{A}(0)})^{-1}$ vanishes at $z=(0,0, \ldots)$. Property $\left(P_{2}\right)$ with

$$
f(\zeta)=1+\frac{1}{2} \zeta-\frac{1}{2 \cdot 4} \zeta^{2}+\frac{1 \cdot 3}{2 \cdot 4 \cdot 6} \zeta^{3}+\cdots=(1+\zeta)^{1 / 2}, \quad|\zeta|<1
$$


implies that $\mathbf{A}=\mathbf{C}^{2}$, where $\mathbf{C}=f(\mathbf{E})=\mathbf{C}^{*}$.

Similarly, if $\mathbf{A}$ is positive, then $\mathbf{A}(0)$ is also positive, but the converse statement need not hold. Take for instance $n=1$ and $\mathbf{A}(z)=z_{1}$. Then it is readily seen that one cannot find $\mathbf{r} \in \mathfrak{R}$ such that $z_{1}=\mathbf{r}^{*}(z) \mathbf{r}(z)$.

We define the ring of polynomials with coefficients in $\mathfrak{R}$ by $\mathfrak{R}[\lambda]$. To avoid confusion between the variable $\lambda$ and the variables $z$ we introduce the notation

$$
\mathscr{I}(\mathbf{r})=\mathbf{r}(0), \quad \mathbf{r} \in \mathfrak{R} .
$$

Definition 3.3. A rational function with values in $\mathfrak{R}^{n \times m}$ is an expression of the form

$$
\mathbf{R}(\lambda)=\mathbf{p}(\lambda)(\mathbf{q}(\lambda))^{-1}
$$

where $\mathbf{p} \in(\mathfrak{R}[\lambda])^{n \times m}$, and $\mathbf{q} \in \mathfrak{R}[\lambda]$ and such that $\mathscr{I}(\mathbf{q}(\lambda)) \not \equiv 0$.

Let $\mathbf{R} \in \mathfrak{R}^{n \times m}(\lambda)$. Then, $\mathscr{I}(\mathbf{R}) \in \mathbb{C}^{n \times m}(\lambda)$, and it is readily seen that

$$
(\mathscr{I}(\mathbf{R}))(\lambda)=\mathscr{I}(\mathbf{R}(\lambda)) \text {. }
$$

It is proved in [6] that every rational function with values matrices with entries in $\mathfrak{R}$ and for which $\mathscr{I}(\mathbf{q}(0)) \neq 0$ can be written as (1.7).

Example 3.4. Let $\mathbf{r} \in \mathfrak{R}$. The function

$$
F_{\mathbf{r}}(\lambda)=(\lambda-\mathbf{r})\left(1-\lambda \mathbf{r}^{*}\right)^{-1} \in \mathfrak{R}(\lambda)
$$

is rational. It is defined for $\lambda \in \mathbb{C}$ such that $1 \neq \lambda(\mathscr{I}(\mathbf{r}))^{*}$.

The next example of rational function need not be defined for $\lambda=0$.

Example 3.5. Let $\mathbf{r} \in \mathfrak{R}$. The function

$$
F_{\mathbf{r}}(\lambda)=(\lambda-\mathbf{r})\left(\lambda-\mathbf{r}^{*}\right)^{-1} \in \mathfrak{R}(\lambda)
$$

is rational. It is defined for $\lambda \in \mathbb{C}$ such that $\lambda \neq(\mathscr{I}(\mathbf{r}))^{*}$.

\section{Analytic functions With Values in $\mathfrak{R}$}

It is possible to define analytic functions with values in a locally convex topological vector space (see for instance the discussion in [13, 14, 17, 16]). Here the structure of $\mathfrak{R}$ allows us to focus, locally, on the classical definition of Hilbert space valued functions, as we now explain.

Proposition 4.1. Let $\Omega \subset \mathbb{C}$ be an open set and let $\mathbf{f}: \Omega \rightarrow \mathfrak{R}$ be a continuous function. Then, $\mathbf{f}$ is locally Hilbert space valued, that is, for every $\zeta_{0} \in \Omega$, there is a compact neighborhood $K$ of $\zeta_{0}$ and a number $p_{0}$ such that $\mathbf{f}(K) \subset I\left(\mathcal{H}_{p_{0}}^{\prime}\right)$. 
Proof: Every $\zeta_{0} \in \Omega$ has a neighborhood $K$ of the form $\overline{B_{\delta}}=\{\zeta \in$ $\left.\Omega ;\left|\zeta_{0}-\zeta\right| \leq \delta\right\}$ for some $\delta>0$. Since $\bar{B}_{\delta}$ is a compact set and $\mathbf{f}$ is continuous, $\mathbf{f}\left(\overline{B_{\delta}}\right)$ is compact in $\mathfrak{R}$, and therefore strongly bounded. See [12, p. 54]. Thus there exists $p_{0} \in \mathbb{N}$ such that $\mathbf{f}\left(\overline{B_{\delta}}\right) \in I\left(\mathcal{H}_{p_{0}}^{\prime}\right)$ and is bounded in the norm of $I\left(\mathcal{H}_{p_{0}}^{\prime}\right)$. See [12, Section 5.3 p. 45].

Therefore we can define an analytic function from $\Omega$ to $\mathfrak{R}$ as a continuous function which locally admits a power expansion with coefficients in one of the spaces $I\left(\mathcal{H}_{p}^{\prime}\right)$. The following example shows that we cannot expect to have a fixed $p$ in general.

Example 4.2. Let $\mathbf{f}(\lambda, z)=\sum_{n=1}^{\infty} n^{\frac{\lambda}{2}} z_{n}$. Then $\mathbf{f}$ is continuous (as a function of $\lambda$ ) from $\mathbb{C}$ into $\mathfrak{R}$, but there is no $p$ such that $\mathbf{f}(\lambda, z)$ (viewed now as a function of $z$ ) belongs to $I\left(\mathcal{H}_{p}^{\prime}\right)$ for all $\lambda \in \mathbb{C}$.

Indeed, let $\lambda_{0} \in \mathbb{C}$. We have

$$
\left(\left\|\mathbf{f}\left(\lambda_{0}\right)\right\|_{p}^{\prime}\right)^{2}=\sum_{n=1}^{\infty}\left|n^{\lambda_{0}}\right|(2 n)^{-p}=2^{-p} \sum_{n=1}^{\infty} n^{\operatorname{Re} \lambda_{0}-p}<\infty,
$$

for $p>\operatorname{Re} \lambda_{0}+1$. To show continuity at a point $\lambda_{0} \in \mathbb{C}$, we take $p>\left|\lambda_{0}\right|+2$, and restrict $\lambda$ to be such that $\left|\lambda_{0}-\lambda\right|<1$. Using the elementary estimate

$$
\left|e^{z_{1}}-e^{z_{2}}\right| \leq\left|z_{1}-z_{2}\right| \cdot \max _{z \in\left[z_{1}, z_{2}\right]}\left|e^{z}\right|
$$

for $z_{1}, z_{2} \in \mathbb{C}$, we have for $n=2,3, \ldots$

$$
\left|n^{\frac{\lambda}{2}}-n^{\frac{\lambda_{0}}{2}}\right| \leq \ln n \frac{\left|\lambda-\lambda_{0}\right|}{2} e^{\frac{\left|\lambda_{0}\right|+1}{2} \ln n}
$$

and so

$$
\begin{aligned}
\left(\left\|\mathbf{f}(\lambda)-\mathbf{f}\left(\lambda_{0}\right)\right\|_{p+2}^{\prime}\right)^{2} & =2^{-p-2} \sum_{n=2}^{\infty}\left|n^{\frac{\lambda}{2}}-n^{\frac{\lambda_{0}}{2}}\right|^{2} n^{-p-2} \\
& \leq \frac{\left|\lambda-\lambda_{0}\right|^{2}}{4} \sum_{n=2}^{\infty} \frac{(\ln n)^{2}}{n^{2}} n^{\left|\lambda_{0}\right|+1-p}
\end{aligned}
$$

and hence the continuity at the point $\lambda_{0}$ in the norm $\|\cdot\|_{p+2}^{\prime}$, and hence in $\mathfrak{R}$. See in particular [12, p. 57] for the latter.

Recall that, in the case of Hilbert space, weak and strong analyticity are equivalent, and can be expressed in terms of power series expansions. The argument uses the uniform boundedness theorem. See [24, Theorem VI.4, p. 189]. We define the evaluation of an $\mathfrak{R}$-valued analytic 
function at a point $\mathbf{r} \in \mathfrak{R}$. We first introduce

$$
\mathfrak{R}_{\Omega}=\{\mathbf{r} \in \mathfrak{R} ; \mathscr{I}(\mathbf{r}) \in \Omega\},
$$

where $\Omega \subset \mathbb{C}$ is open.

Theorem 4.3. Let $\Omega$ be an open subset of $\mathbb{C}$, and let $\mathbf{f}: \Omega \rightarrow \Re$ be an analytic function. Let $\mathbf{r} \in \mathfrak{R}_{\Omega}$, and let

$$
\mathbf{f}(\zeta)=\sum_{n=0}^{\infty} \mathbf{f}_{n}(\zeta-\mathscr{I}(\mathbf{r}))^{n}
$$

be the Taylor expansion around $\mathscr{I}(\mathbf{r}) \in \Omega$, where the $\mathbf{f}_{n} \in \mathcal{H}_{p_{0}}^{\prime}$ for some $p_{0} \in \mathbb{N}$, and where the convergence is in $\mathcal{H}_{p_{0}}^{\prime}$. The series

$$
\mathbf{f}(\mathbf{r})=\sum_{n=0}^{\infty} \mathbf{f}_{n}(\mathbf{r}-\mathscr{I}(\mathbf{r}))^{n}
$$

converges in $\mathcal{H}_{q}^{\prime}$ for some $q>p_{0}$.

Proof: Let $K$ be a compact neighborhood of $\mathscr{I}(\mathbf{r})$, and let $p_{0} \in \mathbb{N}$ be such that $\mathbf{f}(K) \subset \mathcal{H}_{p_{0}}^{\prime}$. Let furthermore $R$ be the radius of convergence of the $\mathcal{H}_{p_{0}}^{\prime}$-valued power series (4.2). In view of (2.11), there exists $p$, which we can assume strictly larger than $p_{0}$, such that

$$
A(2)\|\mathbf{r}-\mathscr{I}(\mathbf{r})\|_{p}^{\prime}<R .
$$

On the other hand, using (2.14), we obtain

$$
\begin{aligned}
\left\|\mathbf{f}_{n}(\mathbf{r}-\mathscr{I}(\mathbf{r}))^{n}\right\|_{p+2}^{\prime} & \leq A(2)\left\|\mathbf{f}_{n}\right\|_{p_{0}}^{\prime}\left\|(\mathbf{r}-\mathscr{I}(\mathbf{r}))^{n}\right\|_{p+2}^{\prime} \\
& \leq\left\|\mathbf{f}_{n}\right\|_{p_{0}}^{\prime}\left(A(2)\|\mathbf{r}-\mathscr{I}(\mathbf{r})\|_{p}^{\prime}\right)^{n}
\end{aligned}
$$

In view of (4.4), the series

$$
\sum_{n=0}^{\infty}\left\|\mathbf{f}_{n}\right\|_{p_{0}}^{\prime}\left(A(2)\|\mathbf{r}-\mathscr{I}(\mathbf{r})\|_{p}^{\prime}\right)^{n}
$$

converges and so the series

$$
\sum_{n=0}^{\infty} \mathbf{f}_{n}(\mathbf{r}-\mathscr{I}(\mathbf{r}))^{n}
$$

converges absolutely in $I\left(\mathcal{H}_{p+2}^{\prime}\right)$.

The evaluation of $\mathbf{f}$ at $\mathbf{r}$ is defined to be $\mathbf{f}(\mathbf{r})$ given by (4.3).

Proposition 4.4. We can rewrite the evaluation at $\mathbf{r}$ as a Cauchy integral

$$
\mathbf{f}(\mathbf{r})=\frac{1}{2 \pi i} \oint \frac{\mathbf{f}(\zeta)}{\zeta-\mathbf{r}} d \zeta
$$


where the integration is along a circle centered at $\mathscr{I}(\mathbf{r})$ and of radius $r<R$ and in $\Omega$.

Proof: As in Theorem 4.3 we consider a compact neighborhood $K$ of $\mathscr{I}(\mathbf{r})$, and let $p_{0}$ be such that that $\mathbf{f}(K) \subset \mathcal{H}_{p_{0}}^{\prime}$. We consider a simple closed path around $\mathscr{I}(\mathbf{r})$ which lies inside $K$.

We have

$$
\begin{aligned}
\frac{1}{2 \pi i} \oint \frac{\mathbf{f}(\zeta)}{\zeta-\mathbf{r}} d \zeta & =\frac{1}{2 \pi i} \oint \frac{\mathbf{f}(\zeta)}{\zeta-\mathscr{I}(\mathbf{r})+\mathscr{I}(\mathbf{r})-\mathbf{r}} d \zeta \\
& =\frac{1}{2 \pi i} \oint \frac{\mathbf{f}(\zeta)}{\zeta-\mathscr{I}(\mathbf{r})}\left\{\sum_{n=0}^{\infty}\left(\frac{\mathbf{r}-\mathscr{I}(\mathbf{r})}{\zeta-\mathscr{I}(\mathbf{r})}\right)^{n}\right\} d \zeta \\
& =\frac{1}{2 \pi i} \sum_{n=0}^{\infty}(\mathbf{r}-\mathscr{I}(\mathbf{r}))^{n}\left\{\oint \frac{\mathbf{f}(\zeta)}{(\zeta-\mathscr{I}(\mathbf{r}))^{n+1}} d \zeta\right\},
\end{aligned}
$$

where we have used the estimates as in the proof of Theorem 4.3 and the dominated convergence theorem to justify the interchange of integration and summation.

Recall that a function $f$ analytic and contractive in the open unit disk is called a Schur function. Furthermore, by the maximum modulus principle, $f$ is in fact strictly contractive in $\mathbb{D}$, unless it is identically equal to a unitary constant. We will call a function $\mathbf{f}$ analytic from the open unit disk $\mathbb{D}$ into $\mathfrak{R}$ a Schur function (notation: $\mathbf{f} \in S_{\mathfrak{R}}$ ) if the function

$$
\lambda \mapsto \mathscr{I}(\mathbf{f}(\lambda))
$$

is a Schur function. For instance, both $1+z_{1} z_{3}$ and $0.5+10 z_{1}-3 z_{5}$ are Schur functions. We now define the analog of the open unit disk by

$$
\mathfrak{R}_{\mathbb{D}}=\{\mathbf{r} \in \mathfrak{R} ; \mathscr{I}(\mathbf{r}) \in \mathbb{D}\},
$$

and the analog of strictly contractive Schur functions as the set of analytic functions $\mathbf{f}: \mathbb{D} \rightarrow \mathfrak{R}$ such that the function $\lambda \mapsto \mathscr{I}(\mathbf{f}(\lambda))$ is a strictly contractive Schur function.

Theorem 4.5. $\mathbf{f} \in S_{\mathfrak{R}}$ is a strictly contractive Schur function if and only if $\mathbf{f}: \mathbb{D} \rightarrow \mathfrak{R}_{\mathbb{D}}$ is analytic.

Proof: If $\mathbf{f}$ is analytic from $\mathbb{D}$ into $\mathfrak{R}$, and such that the $\lambda \mapsto \mathscr{I}(\mathbf{f}(\lambda))$ is a strictly contractive Schur function, it means by definition that the range of $\mathbf{f}$ lies inside $\mathfrak{R}_{\mathbb{D}}$. Conversely, let $\mathbf{f}: \mathbb{D} \rightarrow \mathfrak{R}$ be analytic and such that $\mathscr{I}(\mathbf{f})$ is a strictly contractive Schur function. Then for every $0<r<1$, there exists $k \in \mathbb{N}$ (which may depend on $r$ ) such that $\mathbf{f}(|\lambda| \leq r) \subset I\left(\mathcal{H}^{\prime}{ }_{k}\right)$. We can write $\mathbf{f}$ as 


$$
\mathbf{f}(\lambda)=\sum_{n=0}^{\infty} \lambda^{n} \mathbf{f}_{\mathbf{n}},
$$

where $|\lambda|<r$ and $\mathbf{f}_{\mathbf{n}} \in I\left(\mathcal{H}_{k}^{\prime}\right)$. Now $\mathscr{I}(\mathbf{f})(\lambda)=\sum_{n=0}^{\infty} \lambda^{n} \mathscr{I}\left(\mathbf{f}_{\mathbf{n}}\right)$ for $|\lambda|<r$. Since this holds for all $r \in(0,1)$ the function $\lambda \mapsto \mathbf{f}(\lambda)$ has range inside $\mathfrak{R}_{\mathbb{D}}$.

\section{NevanlinNa-PiCK Interpolation}

In this section we solve the following interpolation problem $(I P)$.

Problem 5.1. Given $n \in \mathbb{N}$ and points $\mathbf{a}_{1}, \ldots, \mathbf{a}_{n}, \mathbf{b}_{1}, \ldots, \mathbf{b}_{n} \in \mathfrak{R}_{\mathbb{D}}$, find all Schur functions $\mathbf{f}$ with coefficients in $\mathfrak{R}$ such that $\mathbf{f}\left(\mathbf{a}_{i}\right)=\mathbf{b}_{i}$ for $i=1,2, \ldots, n$.

The solution of this problem under the assumption that some matrix is strictly positive, is presented in Theorem 5.3 below. We first give some preliminary arguments, and note that if $\mathbf{f}$ is a solution of the interpolation problem 5.1, then $f=\mathscr{I}(\mathbf{f})$ is a solution of the classical interpolation problem

$$
f\left(a_{i}\right)=b_{i}, \quad i=1, \ldots, n,
$$

where we have set

$$
a_{i}=\mathscr{I}\left(\mathbf{a}_{i}\right) \quad \text { and } \quad b_{i}=\mathscr{I}\left(\mathbf{b}_{i}\right), \quad i=1, \ldots n .
$$

This last problem is solved as follows: let $P$ denote the $n \times n$ Hermitian matrix with $i j$ entry equal to

$$
\frac{1-b_{i} b_{j}^{*}}{1-a_{i} a_{j}^{*}} .
$$

A necessary and sufficient condition for (5.5) to have a solution in the family of Schur functions is that $P \geq 0$. We will assume $P>0$. Set, in the notation of the introduction,

$$
\begin{aligned}
A_{\zeta}^{*} & =A=\operatorname{diag}\left(a_{1}^{*}, a_{2}^{*} \ldots, a_{n}^{*}\right), \\
-\left(\begin{array}{c}
B_{+} \\
B_{-}
\end{array}\right) & =\left(\begin{array}{cccc}
1 & 1 & \cdots & 1 \\
b_{1}^{*} & b_{2}^{*} & \cdots & b_{n}^{*}
\end{array}\right) \stackrel{\text { def }}{=} C, \\
J & =\left(\begin{array}{cc}
1 & 0 \\
0 & -1
\end{array}\right) .
\end{aligned}
$$

Furthermore, specializing the formula for $\Theta$ given in the introduction with $z_{0}=1$, or using the formula arising from the theory of reproducing 
kernel Hilbert spaces (see [11, [1]), set

$\Theta(\lambda)=I_{2}-(1-\lambda) C\left(I_{n}-\lambda A\right)^{-1} P^{-1}(I-A)^{-*} C^{*} J \stackrel{\text { def. }}{=}\left(\begin{array}{ll}a(\lambda) & b(\lambda) \\ c(\lambda) & d(\lambda)\end{array}\right)$.

We now gather the main properties of the matrix-valued function $\Theta$ relevant to the present work. For proofs, we refer to [1], [10], [11].

Proposition 5.2. The following hold:

(a) The function $\Theta$ is J-inner with respect to the open unit disk.

(b) $\Theta$ has no poles in $\mathbb{D}$ and $a(\lambda) \sigma+b(\lambda) \neq 0$ for all $\lambda \in \mathbb{D}$ and all $\sigma$ in the closed unit disk.

(c) The identity

$$
\left(\begin{array}{ll}
1 & -b_{i}
\end{array}\right) \Theta\left(a_{i}\right)=0, \quad i=1, \ldots n .
$$

is valid. $(d)$ The linear fractional transformation

$$
T_{\Theta(\lambda)}(\sigma(\lambda)) \stackrel{\text { def. }}{=} \frac{a(\lambda) \sigma(\lambda)+b(\lambda)}{c(\lambda) \sigma(\lambda)+d(\lambda)}
$$

describes the set of all solutions of the problem (5.5) in the family of Schur functions when $\sigma$ varies in the family of Schur functions.

To solve the interpolation problem 5.1 we introduce the matrices $\mathbf{A}, \mathbf{C}$ and $\mathbf{P}$, with entries in $\mathfrak{R}$, built by formulas (5.6) and (5.7), but with $\mathbf{a}_{1}, \ldots, \mathbf{a}_{n}, \mathbf{b}_{1}, \ldots, \mathbf{b}_{n}$ instead of $a_{1}, \ldots, a_{n}, b_{1}, \ldots, b_{n}$. Note that $\mathbf{P}>0$ since $P>0$, and we can define the $\mathfrak{R}^{2 \times 2}$-valued function $\Theta$ as $\Theta$ but with $\mathbf{A}, \mathbf{C}$ and $\mathbf{P}$ instead of $A, C$ and $P$. We have

$$
\mathscr{I}(\mathbf{A})=A, \quad \mathscr{I}(\mathbf{C})=C, \quad \text { and } \quad \mathscr{I}(\mathbf{P})=P .
$$

Furthermore,

$$
\mathscr{I}(\Theta(\lambda))=\Theta(\lambda)
$$

Theorem 5.3. Assume $\mathbf{P}>0$. Then, there is a one-to-one correspondence between the solutions $\mathbf{f}$ of the problem $5.1 \mathrm{in} S_{\mathfrak{R}}$ and the elements $\mathbf{g} \in S_{\mathfrak{R}}$ via the linear fractional transformation $\mathbf{f}=T_{\mathbf{\Theta}}(\mathbf{g})$.

Proof: We first claim that the matrix-valued function $\Theta$ satisfies the counterparts of (5.8), that is,

$$
\left(1-\mathbf{b}_{i}\right) \boldsymbol{\Theta}\left(\mathbf{a}_{i}\right)=0, \quad i=1, \ldots n .
$$

This is done using the permanence of algebraic identities. See [9, p. 456] for the latter. Indeed, the function

$$
\left(\operatorname{det}\left(I_{n}-\lambda A\right)\right)\left(\operatorname{det}\left(I_{n}-A^{*}\right)\right)(\operatorname{det} P)\left(\prod_{\ell, j=1, \ldots n}\left(1-a_{\ell} a_{j}^{*}\right)\right) \Theta(\lambda)
$$


is a polynomial in $\lambda$ with coefficients which are themselves polynomials in the $a_{i}$ and the $b_{j}$, with entire coefficients. Therefore, multiplying both sides of (5.8) by the polynomial function

$$
\left(\operatorname{det}\left(I_{n}-\lambda A\right)\right)\left(\operatorname{det}\left(I_{n}-A^{*}\right)\right)(\operatorname{det} P)\left(\prod_{\ell, j=1, \ldots n}\left(1-a_{\ell} a_{j}^{*}\right)\right)
$$

evaluated at $\lambda=a_{i}(i=1,2, \ldots, n)$, and taking the real and imaginary part of the equalities (5.8), we obtain for each $i$ four polynomial identities in the $4 n$ real variables $\operatorname{Re} a_{j}, \operatorname{Re} b_{j}, \operatorname{Im} a_{j}, \operatorname{Im} b_{j}$, with $j=1, \ldots n$, with entire coefficients, namely

$\operatorname{Re}\left\{\left(1-b_{i}\right) \operatorname{det}\left(I-a_{i} A\right) \operatorname{det}\left(I-A^{*}\right) \operatorname{det} P \prod_{\ell, j=1, \ldots n}\left(1-a_{\ell} a_{j}^{*}\right) \Theta\left(a_{i}\right)\right\}=$

$$
=\left(\begin{array}{ll}
0 & 0
\end{array}\right) \text {, }
$$

$\operatorname{Im}\left\{\left(1-b_{i}\right) \operatorname{det}\left(I-a_{i} A\right) \operatorname{det}\left(I-A^{*}\right) \operatorname{det} P \prod_{\ell, j=1, \ldots n}\left(1-a_{\ell} a_{j}^{*}\right) \Theta\left(a_{i}\right)\right\}=$

$$
=\left(\begin{array}{ll}
0 & 0
\end{array}\right) .
$$

It follows (see [9, p. 456]) that these identities hold in any commutative rings, and in particular in $\mathfrak{R}$ :

$\operatorname{Re}\left\{\left(1-\mathbf{b}_{i}\right) \operatorname{det}\left(I-\mathbf{a}_{i} \mathbf{A}\right) \operatorname{det}\left(I-\mathbf{A}^{*}\right) \operatorname{det} \mathbf{P} \prod_{\ell, j=1, \ldots n}\left(1-\mathbf{a}_{\ell} \mathbf{a}_{j}^{*}\right) \boldsymbol{\Theta}\left(\mathbf{a}_{i}\right)\right\}=$

$$
=\left(\begin{array}{ll}
0 & 0
\end{array}\right) \text {, }
$$

$\operatorname{Im}\left\{\left(1-\mathbf{b}_{i}\right) \operatorname{det}\left(I-\mathbf{a}_{i} \mathbf{A}\right) \operatorname{det}\left(I-\mathbf{A}^{*}\right) \operatorname{det} \mathbf{P} \prod_{\ell, j=1, \ldots n}\left(1-\mathbf{a}_{\ell} \mathbf{a}_{j}^{*}\right) \boldsymbol{\Theta}\left(\mathbf{a}_{i}\right)\right\}=$

$$
=\left(\begin{array}{ll}
0 & 0
\end{array}\right) .
$$

We now use the fact that we are in the ring $\mathfrak{R}$. Because of the choice of the $\mathbf{a}_{j}$, the element

$$
\operatorname{det}\left(I-\mathbf{a}_{i} \mathbf{A}\right) \operatorname{det}\left(I-\mathbf{A}^{*}\right) \prod_{\ell, j=1, \ldots n}\left(1-\mathbf{a}_{\ell} \mathbf{a}_{j}^{*}\right)
$$

is invertible in $\mathfrak{R}$. When furthermore $\mathbf{P}>0$ we can divide both sides of the above equalities by

$$
\operatorname{det}\left(I-\mathbf{a}_{i} \mathbf{A}\right) \operatorname{det}\left(I-\mathbf{A}^{*}\right) \prod_{\ell, j=1, \ldots n}\left(1-\mathbf{a}_{\ell} \mathbf{a}_{j}^{*}\right) \operatorname{det} \mathbf{P}
$$

and obtain (5.10). 
Let now $\mathbf{r} \in S_{\Re}$, and let $\mathbf{u}, \mathbf{v}$ be analytic $\Re$-valued functions defined by

$$
\left(\begin{array}{c}
\mathbf{u}(\lambda) \\
\mathbf{v}(\lambda)
\end{array}\right)=\boldsymbol{\Theta}(\lambda)\left(\begin{array}{c}
\mathbf{r}(\lambda) \\
1
\end{array}\right)=\left(\begin{array}{c}
\mathbf{a}(\lambda) \mathbf{r}(\lambda)+\mathbf{b}(\lambda) \\
\mathbf{c}(\lambda) \mathbf{r}(\lambda)+\mathbf{d}(\lambda)
\end{array}\right)
$$

Using (5.10) we have that

$$
\mathbf{u}\left(\mathbf{a}_{i}\right)=\mathbf{b}_{i} \mathbf{v}\left(\mathbf{a}_{i}\right), \quad i=1, \ldots, n .
$$

To conclude, we need to show that $\mathbf{v}\left(\mathbf{a}_{i}\right)$ is invertible in $\mathfrak{R}$ for $i=$ $1, \ldots n$. But we have

$$
\mathscr{I}\left(\mathbf{v}\left(\mathbf{a}_{i}\right)\right)=c\left(a_{i}\right) \mathscr{I}(\mathbf{r})\left(a_{i}\right)+d\left(a_{i}\right), \quad i=1, \ldots, n .
$$

Since the function $\Theta(\lambda)=\mathscr{I}(\Theta(\lambda))$ is $J$-unitary on the unit circle and has no poles there. Therefore, we have $c\left(a_{i}\right) \mathscr{I}(\mathbf{r})\left(a_{i}\right)+d\left(a_{i}\right) \neq 0$ (see item $(b)$ in Proposition 5.2), and hence $\mathbf{v}\left(\mathbf{a}_{i}\right)$ is invertible in $\mathfrak{R}$. Therefore $\mathbf{u v}^{-1}=T_{\boldsymbol{\Theta}}(\mathbf{r})$ is a solution of the interpolation problem.

Assume now that $\mathbf{f}$ is a solution. Then, we know from the discussion before the theorem that there exists a Schur function $\sigma(\lambda)$ such that

$$
\mathscr{I}(\mathbf{f}(\lambda))=T_{\mathscr{I}(\boldsymbol{\Theta}(\lambda))}(\sigma(\lambda)) .
$$

Define a $\mathfrak{R}$-valued function $\mathbf{r}$ by

$$
\mathbf{f}(\lambda)=T_{\mathbf{\Theta}(\lambda)}(\mathbf{r}(\lambda)) .
$$

Taking $\mathscr{I}$ on both sides of this expression we obtain

$$
\mathscr{I}(\mathbf{f}(\lambda))=T_{\mathscr{I}(\Theta(\lambda))}(\mathscr{I}(\mathbf{r}(\lambda))) .
$$

Comparing with (5.11), we obtain $\mathscr{I}(\mathbf{r}(\lambda))=\sigma(\lambda)$, and hence $\mathbf{r} \in S_{\Re}$.

\section{More General interpolation PROBlem}

The matrix-valued function $\Theta$ defined by (1.3) and describing the set of solutions of the bitangential problem satisfies the conditions

$$
\begin{aligned}
& \sum_{\lambda_{0} \in \mathbb{D}} \operatorname{Res}_{\lambda=\lambda_{0}}\left(\left(\lambda I-A_{\zeta}\right)^{-1} B_{+} B_{-}\right) \Theta(\lambda)=0 \\
& \sum_{\lambda_{0} \in \mathbb{D}} \operatorname{Res}_{\lambda=\lambda_{0}} \Theta\left(1 / \lambda^{*}\right)^{*}\left(\begin{array}{c}
C_{-}\left(\lambda I-A_{\pi}\right)^{-1} \\
C_{+}
\end{array}\right)=0 .
\end{aligned}
$$

See also 4]. As for the Nevanlinna-Pick case, these conditions can be translated into a finite number of polynomial equations with coefficients in $\mathbb{Z}$, and the principle of permanence of identities allows to extend these properties in the case of a commutative ring. On the other hand, we do not know how to extend the third interpolation property, 
and so the method is not applicable to the most general bitangential interpolation problem. On the other hand, if we restrict the parameter to be a constant contractive matrix, the third condition also translates into a polynomial identity with entire coefficents, and the same method can still be used. The case of functions with poles inside the open unit disk, or the degenerate cases, are more difficult to treat, and will be considered elsewhere.

\section{REFERENCES}

[1] D. Alpay. The Schur algorithm, reproducing kernel spaces and system theory. American Mathematical Society, Providence, RI, 2001. Translated from the 1998 French original by Stephen S. Wilson, Panoramas et Synthèses. [Panoramas and Syntheses].

[2] D. Alpay, H. Attia, and D. Levanony. Une généralisation de l'intégrale stochastique de Wick-Itô. C. R. Math. Acad. Sci. Paris, 346(5-6):261-265, 2008.

[3] D. Alpay, H. Attia, and D. Levanony. On the characteristics of a class of gaussian processes within the white noise space setting. Stochastic processes and applications, 120:1074-1104, 2010.

[4] D. Alpay, P. Bruinsma, A. Dijksma, and H.S.V. de Snoo. Interpolation problems, extensions of symmetric operators and reproducing kernel spaces II. Integral Equations Operator Theory, 14:465-500, 1991.

[5] D. Alpay and D. Levanony. Linear stochastic systems: a white noise approach. Acta Applicandae Mathematicae, 110:545-572, 2010.

[6] D. Alpay, D. Levanony, and A. Pinhas. Linear stochastic state space theory in the white noise space setting. SIAM Journal of Control and Optimization, 48:5009-5027, 2010.

[7] D. Alpay and Guy Salomon. A family of commutative rings with a Våge's inequality. In preparation.

[8] Daniel Alpay and David Levanony. Linear stochastic systems: a white noise approach. Acta Appl. Math., 110(2):545-572, 2010.

[9] Michael Artin. Algebra. Prentice Hall Inc., Englewood Cliffs, NJ, 1991.

[10] J. Ball, I. Gohberg, and L. Rodman. Interpolation of rational matrix functions, volume 45 of Operator Theory: Advances and Applications. Birkhäuser Verlag, Basel, 1990.

[11] H. Dym. J-contractive matrix functions, reproducing kernel Hilbert spaces and interpolation. Published for the Conference Board of the Mathematical Sciences, Washington, DC, 1989.

[12] I.M. Gelfand and G.E. Shilov. Generalized functions. Volume 2. Academic Press.

[13] A. Grothendieck. Sur certains espaces de fonctions holomorphes. I. J. Reine Angew. Math., 192:35-64, 1953.

[14] A. Grothendieck. Sur certains espaces de fonctions holomorphes. II. J. Reine Angew. Math., 192:78-95, 1953.

[15] I.M. Guelfand and N.Y. Vilenkin. Les distributions. Tome 4: Applications de l'analyse harmonique. Collection Universitaire de Mathématiques, No. 23. Dunod, Paris, 1967. 
[16] M. Hervé. Analytic and plurisubharmonic functions in finite and infinite dimensional spaces. Number 198 in Lecture Notes in Mathematics. SpringerVerlag, 1971.

[17] M. Hervé. Analyticity in infinite-dimensional spaces, volume 10 of de Gruyter Studies in Mathematics. Walter de Gruyter \& Co., Berlin, 1989.

[18] T. Hida, H. Kuo, J. Potthoff, and L. Streit. White noise, volume 253 of Mathematics and its Applications. Kluwer Academic Publishers Group, Dordrecht, 1993. An infinite-dimensional calculus.

[19] T. Hida and Si Si. Lectures on white noise functionals. World Scientific Publishing Co. Pte. Ltd., Hackensack, NJ, 2008.

[20] H. Holden, B. Øksendal, J. Ubøe, and T. Zhang. Stochastic partial differential equations. Probability and its Applications. Birkhäuser Boston Inc., Boston, MA, 1996.

[21] M. Kaashoek. State space theory of rational matrix functions and applications. In P. Lancaster, editor, Lectures on operator theory and its applications, volume 3 of Fields Institute Monographs, pages 235-333. American Mathematical Society, 1996.

[22] R. E. Kalman, P. L. Falb, and M. A. Arbib. Topics in mathematical system theory. McGraw-Hill Book Co., New York, 1969.

[23] Hui-Hsiung Kuo. White noise distribution theory. Probability and Stochastics Series. CRC Press, Boca Raton, FL, 1996.

[24] M. Reed and B. Simon. Methods of modern mathematical physics. I. Functional analysis. Academic Press, New York, 1972.

[25] E.D. Sontag. Linear systems over commutative rings: A survey. Ricerche di Automatica, 7:1-34, 1976.

(DA) Department of Mathematics

Ben Gurion University of the Negev

P.O.B. 653,

BE'ER SHEVA 84105,

ISRAEL

E-mail address: dany@math.bgu.ac.il

(HA) Department of Mathematics

Sami Shamoon College of Engineering

BE'ER SHEVA 84100,

ISRAEL

E-mail address: haima@sce.ac.il 